\title{
Cluster headache due to intranasal herpes simplex: a case report
}

\author{
Leonardo de Sousa Bernardes ${ }^{1}$ (D) Renan Domingues Oliveira' $^{1}$, Mário Fernando Prieto Peres $^{2}$ iD \\ 'Santa Casa de Misericórdia de São Paulo, São Paulo, Brazil. \\ ${ }^{2}$ Hospital Israelita Albert Einstein, São Paulo, Brazil.

\section{Introduction} \\ Cluster headache $(\mathrm{CH})$ is a rare kind of primary headache, accounting to roughly $0.1 \%$ worldwide; it is considered \\ a trigeminal autonomic cephalalgias. Structural diseases may also cause cluster headache; in a recent systematic \\ review, the most common pathologies associated were vascular, tumoral, inflammatory and infection. Intranasal and \\ sinus infections such as sinusitis and mucocele were also reported.
}

\section{Goals}

To describe a case report of a male patient with cluster headache due to intranasal herpes simplex and enhance the discussion about an infectious disease mimicking $\mathrm{CH}$.

\section{Methods}

The data was collected through electronic medical record of the patient and later we performed a review of the literature regarding herpes simplex and cluster headache.

\section{Results}

A 43-year-old right-handed man, presented 2 years ago with a left sided excruciating pain, starting in the orbital region irradiating to the ear and nose, lasting 15 minutes, associated with tearing and ipsilateral rhinorrhea. One year later, he presented again with another cycle of headache with the same features. An otolaryngologist evaluated the patient at the emergency department, and rhinoscopy disclosed lesions in the internal vestibular region of the nose, hyperemia and scars suggestive of herpes simplex. Antibodies $\lg M$ and $\lg G$ for herpes simplex were positive. A diagnosis of nasal herpes was made. Patient started on Valacyclovir $500 \mathrm{mg}$ tid, headaches had prompted relief 3 days after starting the antiviral therapy. In a follow up visit 6 months after the therapy he kept headache free.

\section{Conclusions}

Our report seems to be the first case linking cluster headache to intranasal herpes simplex infection. The presence of $\lg M$ and $\lg G$ class antibodies confirm a chronic infection with recent reactivation, associating the cluster headache with the HSV reactivation process.

Keywords: cluster headache, herpes simplex, secondary cluster. 